4th Tamura Symp. on Accelerator Physics, The Univ. of Texas at Austin, 11/14-16/94.

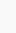

\title{
A Report on the Indiana University Workshop on Future U.S. Hadron Facilities
}

\author{
M. J. Syphers \\ Brookhaven National Laboratory* \\ Upton, NY 11973
}

\begin{abstract}
In July 1994 a workshop was held at Indiana University to study and discuss options for future hadron collider facilities in the United States, and to identify related R\&D programs. The workshop was conducted under the auspices of the Accelerator Physics, Technologies, and Facilities Working Group of the DPF Long Term Planning Study. Roughly 50 participants from 17 institutions in the U.S. and Europe (CERN) were organized into six working groups to study magnets, cryogenics and vacuum, antiproton sources, injectors, interaction regions, and lattice and beam dynamics. Upgrades to existing facilities (namely, Fermilab) and a post-LHC facility were discussed at the workshop. In this paper, the discussion will focus on the post-LHC facility. One of the specific goals of the workshop was to develop a defensible parameters list for a $30 \mathrm{TeV} \times$ $30 \mathrm{TeV}$ hadron collider with luminosity of $1 \times 10^{34} \mathrm{~cm}^{-2} \mathrm{sec}^{-1}$. While this accelerator would have only $50 \%$ higher energy than the SSC design, it was realized that the role of synchrotron radiation at this energy would significantly enhance the design and operation of the machine. Radiation damping times of a few hours, rather than one day, can be realized thus allowing less intense, but brighter proton beams.
\end{abstract}

\section{THE INDIANA WORKSHOP}

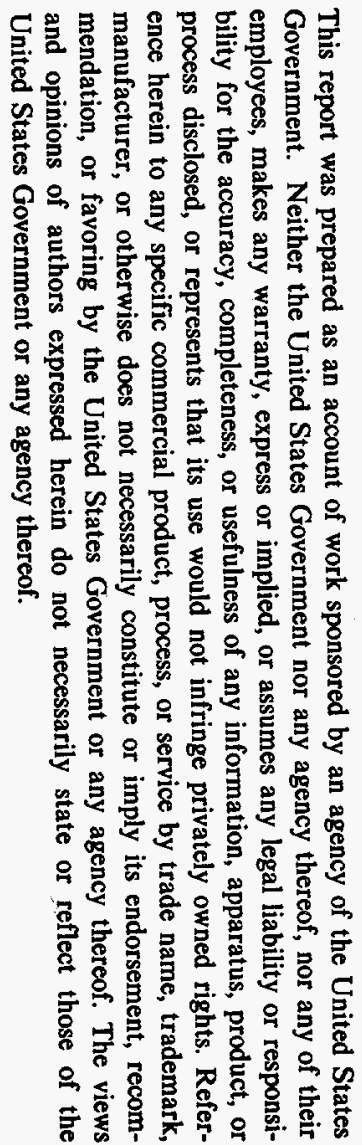

A workshop on "Future Hadron Facilities in the U.S." was held at Indiana University from July 6-10, 1994. This workshop, spurred by the recent termination of the Superconducting Super Collider project[1],[2], was designed to address possible directions for the U.S. HEP program utilizing hadron colliding beams accelerators. The working group operated under the following general charge:

*Work performed under the auspices of the U.S. Dept. of Energy 
"The Accelerator Physics, Technologies, and Facilities Working Group will undertake an assessment of the current state-of-the-art and foreseeable advances in accelerator facilities utilized to support High Energy Physics research into the 21-st century. The group will attempt to identify fundamental performance limitations in existing and proposed facilities, to identify avenues for advancement in the implementing technologies, to assess the promise and prospects of new accelerator directions, and to construct realistic timetables for achievement of specific performance milestones."

At the Indiana Workshop, two specific accelerator models were used in an attempt to focus the work. The first was a $2 \times 2 \mathrm{TeV} \overline{\mathrm{p}}$-p collider with luminosity of $2 \times 10^{33} \mathrm{~cm}^{-2} \mathrm{sec}^{-1}$ operating in the Fermilab Tevatron tunnel. The second accelerator considered was a $30 \times 30 \mathrm{TeV}$ p-p collider, with luminosity of $1 \times 10^{34} \mathrm{~cm}^{-2} \mathrm{sec}^{-1}$. These two facilities were chosen because they were felt to span the potential needs of the U.S. HEP community following the completion of the Main Injector project, through and beyond the period involving the LHC in Europe. It was felt that the R\&D which was spawned by such accelerator designs would provide a strong basis for any direction the community elected to procede in the future. In addition, the workshop participants felt an overwhelming need to quickly draw upon the expertise and experience gained during the design and initial construction of the SSC.

Following some quick remarks on the $2 \times 2 \mathrm{TeV}$ Collider, this paper will be primarily devoted to a discussion of the $30 \times 30 \mathrm{TeV}$ accelerator parameters. A complete overview of the workshop and its conclusions can be found in the Workshop Proceedings.[3]

\section{SOME REMARKS ON A 2 TEV COLLIDER}

One major portion of the workshop was devoted to the development of a defensible parameters list for a $2 \times 2 \mathrm{TeV} \overline{\mathrm{p}}-\mathrm{p}$ collider in the Tevatron tunnel. The major technical issues for this facility are the accumulation rate and storage capacity of the antiprotons. A luminosity of $2 \times 10^{33} \mathrm{~cm}^{-2} \mathrm{sec}^{-1}$ requires an accumulation rate of $9 \times 10^{11} \overline{\mathrm{p}} / \mathrm{hour}$, which is 6 times the rate expected at Fermilab following the Main Injector upgrade. In addition, a storage capacity of up to $10^{13}$ antiprotons is required, which is roughly 3-5 times the present capacity of the Fermilab Antiproton Accumulator ring. Thus, at least one and perhaps two new rings are required for this scenario. In an attempt to maintain the luminosity, but decrease the number of bunches required (hence, alleviating multi-bunch instabilities as well as long range interactions with the other beam), it became obvious that a trade-off between number of bunches and interactions per bunch crossing at the detectors would be an issue. This issue is at the center of the $30 \times 30 \mathrm{TeV}$ collider design as well. In the $2 \times 2$ 


\section{DISCLAIMER}

Portions of this document may be illegible in electronic image products. Images are produced from the best available original document. 
$\mathrm{TeV}$ design, the number of interactions per crossing varied between 2.5 and 17 . Obviously, the interaction rate between the accelerator and detector designers will need to be quite high as well.

Other upgrades at Fermilab, such as a p-p Collider in the Tevatron tunnel[4], site-fillers, etc., were not discussed at the workshop.

\section{A FUTURE LARGE COLLIDER}

We will now focus more on the discussion surrounding a large, SSC-scale hadron facility which would be a conceivable post-LHC instrument. While the energy of the machine considered is only $50 \%$ higher than the energy of the SSC, it was realized that radiation damping in such an accelerator has a significant impact on the operating characteristics and must be utilized to advantage in the design of the facility. The damping time of the transverse emittance of the SSC was roughly 24 hours. It was well understood that this meant that the peak luminosity of the SSC would rise from its initial value to a value some $50 \%$ higher (for the expected initial conditions on emittance, and intra-beam scattering) over a period of 1-2 days. A machine of roughly the same size (assuming higher field magnets, say) but with $50 \%$ higher energy would have damping times of $4-5$ hours with no further modifications to the basic design. This rate can even be enhanced, as will be discussed below.

Fig. 1 shows an estimate of how the emittance, luminosity, bunch intensity, and beam-beam tune shift might behave in such an accelerator. The luminosity rises by a factor of two over a 4 hour period, and slowly falls again in about the same amount of time. The rapid fall in initial emittance implies that the overall integrated luminosity of this accelerator will not depend too strongly on the initial emittance - hence, it should be possible to relax requirements for the injector system, as well as for the injection field quality of the collider.

\section{Parameters}

A partial parameters list of the $30 \mathrm{TeV}$ accelerator is shown in Table 1 . Several of the parameters were massaged extensively during the course of the Workshop. The energy of $30 \mathrm{TeV}$ was chosen purely to illustrate the potential consequences of operating a hadron accelerator in a radiation damping regime. For a fixed field, if the energy is too low, the damping becomes insufficient to be useful. If the energy is too high, then the radiated power becomes prohibitive for the cryogenic magnets. Since the initial emittance can be somewhat relaxed, a choice of 30:1 for the dynamic range of the accelerator was chosen with an allowance for $50 \%$ emittance growth at injection. The dipole field of $12.5 \mathrm{~T}$ represents next generation superconducting magnets, though a range of dipole fields can be accommodated in this approach. Lower fields, and perhaps 
a)

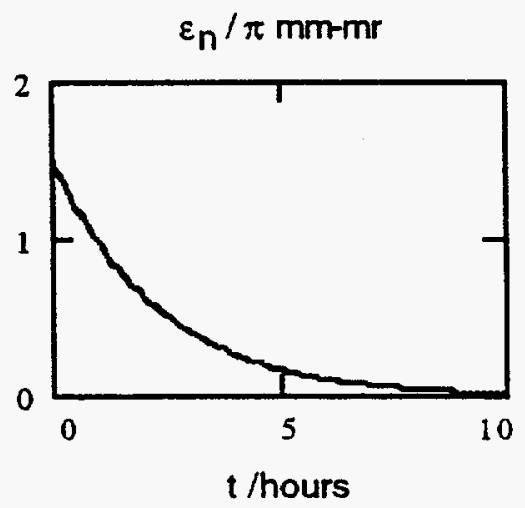

c)

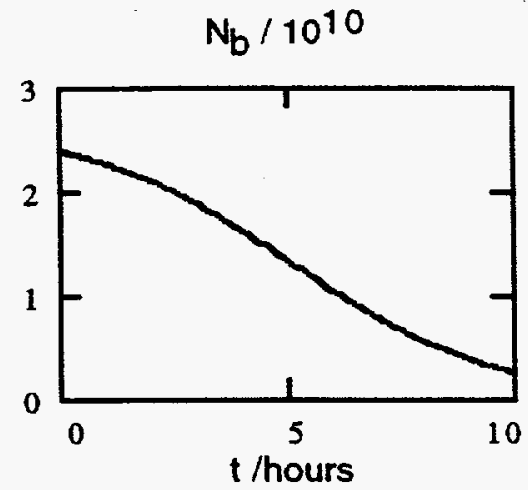

b)

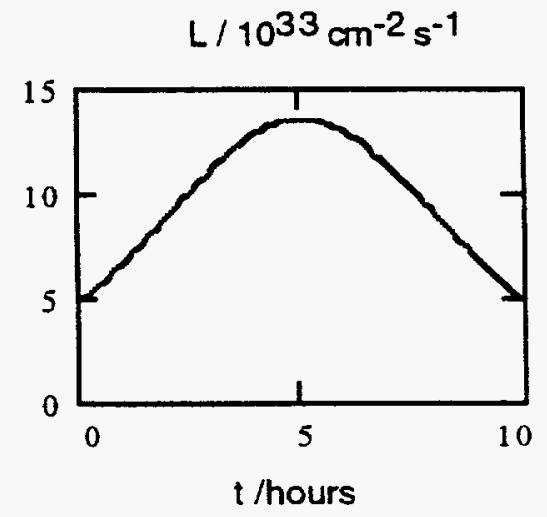

d)

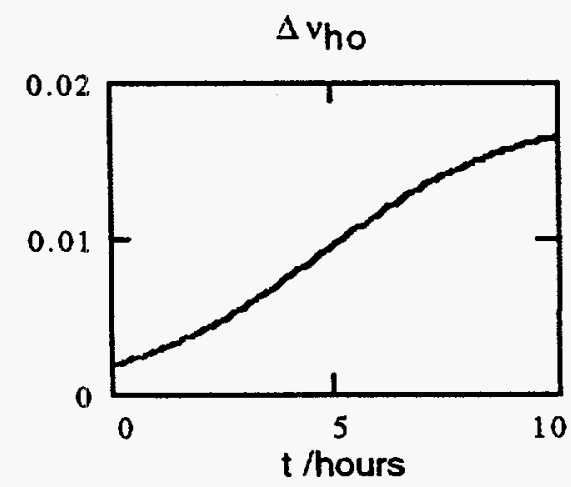

Figure 1: (a) Emittance, (b) luminosity, (c) bunch intensity, and (d) beambeam tune shift vs. time during store. 
Table 1: $30 \mathrm{TeV}$ Collider Parameters

\begin{tabular}{|l|c|}
\hline Parameter & \\
\hline Beam Energy (TeV) & 30 \\
Init. Luminosity ( $\left.\times 10^{34} \mathrm{~cm}^{-2} \mathrm{sec}^{-1}\right)$ & 0.35 \\
& \\
Injection Energy (TeV) & 1 \\
No. of IR's & 2 \\
Dipole Field (T) & 12.5 \\
Circumference (km) & 60 \\
No. of Dipole magnets & 3335 \\
& \\
Init. Trans. Emittance at Coll.(rms, mm-mr) & $1.5 \pi$ \\
Init. Trans. Emittance at Inj. (rms, mm-mr) & $1.0 \pi$ \\
Init. Long. Emittance (rms, eV-s) & 0.5 \\
& \\
RF frequency (MHz) & 360 \\
RF voltage (MV) & 25 \\
Bunch length at Coll. (rms, cm) & 4 \\
& \\
Bunch intensity (1010) & 2.4 \\
No. of bunches & 2030 \\
Bunch spacing (ns) & 100 \\
No. Events per crossing (@60mb, 10 & 60 \\
Rev. frequency (kHz) & 5 \\
Init. beam lifetime - burn off (hr) & 16 \\
Init. $\Delta \nu$ beam-beam head-on / IR & \\
Crossing angle ( $\mu$ rad) & 0.002 \\
Init. $\Delta \nu$ long-range / IR & 50 \\
$\beta^{*}$ (cm) & 0.0039 \\
Syn. Radiation power / length / ring (W/m) & 20 \\
Total Syn. Rad. power / ring (kW) & 0.56 \\
Rad. transverse damping time (hr) & 29 \\
Rad. longitudinal damping time (hr) & 4.7 \\
Stored energy per beam (MJ) & 2.3 \\
& 230 \\
Filling time / ring (min) & \\
Injector cycle time (min) & 10 \\
Acceleration time (min) & 1 \\
Injection dipole field (T) & 5 \\
& 4 \\
\hline
\end{tabular}


non-FODO lattices, can produce similar results with modest remixing of the accelerator parameters.

One of the more controversial issues of this scenario is the bunch spacing and associated number of events per crossing. The total beam intensity was chosen by the desire to optimize the particle "burn-off" rate at a luminosity of $10^{34} \mathrm{~cm}^{-2} \mathrm{sec}^{-1}$ while minimizing the radiated power. An optimum store length for the parameters chosen is about 8 hours. During this time the ratio of average luminosity to peak luminosity is about $70 \%$. The bunch intensity, and hence the bunch spacing and number of bunches was influenced by stability considerations. The projected 60 events per crossing is roughly 3 times the number of events per crossing expected at the LHC. However, this machine would have much more trigger processing time between crossings as well. Much more discussion on this point is to be expected in the future.

\section{Lattice Issues}

Drawing upon the lessons from the SSC, it was felt that simplification of the lattice - from a hardware standpoint - was necessary. This entailed developing a workable lattice in which the number of different types of components are minimized, long cable runs for correctors are avoided and the number of power leads are minimized. This philosophy led to a design with "sparse/lumped" correctors, and assumptions about having power and vacuum hardware physically attached to the same cryostat containing the main quadrupole magnets, thus avoiding the need for separate "spool pieces" in every half-cell.

Discussions on magnet aperture and field quality, and instabilities thresholds were also held. Impedance issues were not seen to be an immediate issue that needed to be addressed in this workshop. The prominent impedance issue will be the beam tube liner, and so will depend very strongly on the optimized magnet and cryogenic/vacuum design.

\section{Standard Arc Modules}

The Lattice Working Group at the workshop investigated ways to simplify the lattice and reduce the cost of the accelerator. The emphasis on much of the discussion was how to avoid the need for "spool pieces," which are devices used in present superconducting accelerators to interface the accelerator to power and vacuum systems, beam instrumentation systems, and which typically contain accelerator correction magnets. In the SSC, each $90 \mathrm{~m}$ half cell of the Collider ring contained a $5 \mathrm{~m}$ spool piece, at least half of which was used for correction magnets - dipoles, quadrupoles, and sextupoles in particular, with occasional other correctors such as skew quadrupoles. There were a variety of spool pieces, some of which contained recooling apparatus, some 


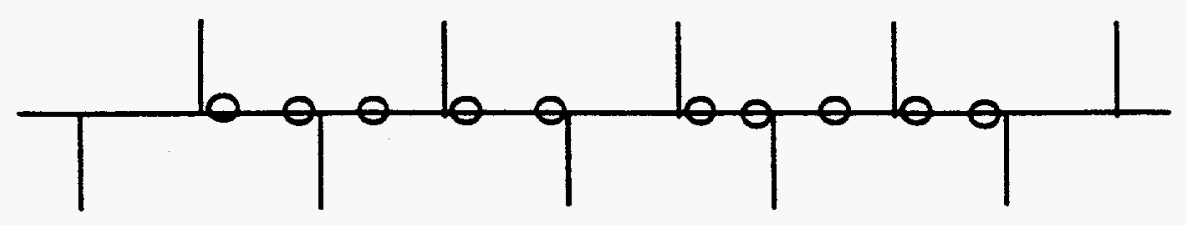

Figure 2: Dispersion-matched free-space regions in otherwise standard FODO cells; the circles indicate locations where dipole magnets are missing from the normal cell structure.

having power and vacuum interfaces, different ones with different corrector packages, etc.

The Working Group envisioned a scenario as follows. The "arcs" of the accelerator are made up of $90^{\circ} \mathrm{FODO}$ cells. Each FODO cell is composed of a quadrupole and 5 dipole magnets. As a working example, the Working Group compared these concepts to the general lay-out of the SSC arcs. In the SSC. roughly every 24 cells there was an interface point to the power and cryogenics systems. In the new scheme, the lattice would contain a section of 4 cells which would have free space generated by leaving out a sequence of dipole magnets (10, in our example) as a "dispersion-matched insertion." These "free spaces" would contain "empty cryostats," " which could then be converted to function as spool pieces as required. That is, there would be devices which are of the same length and outer diameter as standard dipole cryostats, but which may contain correctors, power feeds, cryo feeds. etc. as needed. An example of such an insertion is shown in Fig. 2.

To avoid having spool pieces in each half cell, standard systems hardware, which occurs every cell, would be designed into the quadrupole assembly resulting in a single piece of hardware. It was assumed that the quadrupole stands would be remotely moveable with stepping motors to perform coarse orbit adjustments throughout each arc. In regions such as the IRs and utility straight sections (not discussed by this Working Group), steering correction magnets could be implemented (as well as in the free-space insertions discussed above, as necessary) to perform injection bumps, etc. However, in the arcs, where such distortions generally are not necessary, the quadrupole alignment could be set and left alone. To make a $5 \mathrm{~mm}$ orbit bump in the ring, one would need only about $\pm 1 \mathrm{~mm}$ movement of a standard quadrupole. Such remotely controllable magnet stands could also allow one to relax the alignment requirements of the accelerator upon installation. It was pointed out, however, that if this accelerator incorporates a 2-in-1 magnet design, simultaneous alignment of both beams using moveable magnets would be extremely difficult. In this case, special bore-tube windings could be used to perform steering.

The adjustment of the global tunes of the accelerator will be performed by Phase Trombones - one at each end of each arc. These consist of 5 "stan- 


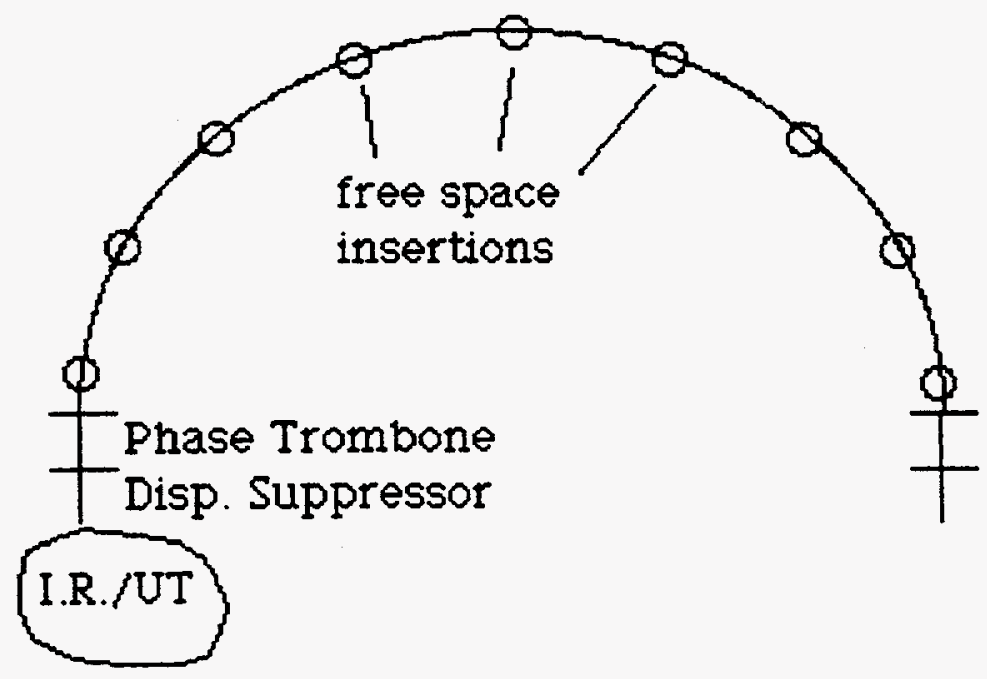

Figure 3: Schematic layout of one arc of the accelerator. The circles indicate locations of the free-space regions (see Fig. 2), where power and cooling utilities are located.

dard" cells with 5 independently controlled quadrupole circuits, allowing one to tune the phase advance across the Trombone in each plane, while keeping the section matched to the rest of the ring. If it is found that such sections cannot meet the required tuning range (roughly 1-2 units), then other measures would need to be considered, with the most straightforward solution being the placement of trim quadrupoles in the "free-space" insertions.

Chromaticity adjustments will be made using sextupoles in the freespace insertions. In our example, each insertion contains four straight sections by $F$ quads, four next to $D$ quads, each the length of a standard dipole. It was felt that this would be plenty of space to encorporate the sextupoles necessary for chromaticity correction. Naturally, the effects on dynamic aperture of such a lumped scheme will have to be studied carefully. If necessary, a systematic $b_{2}$ term in the dipole magnets could be incorporated into the design to compensate the natural chromaticity of the arcs.

In addition, the free-space insertions contain "missing magnets" in the middle of half cells. These can be used to contain skew quadrupoles to perform decoupling of the transverse degrees of freedom.

A schematic layout of one arc of the accelerator is shown in Fig. 3. 


\section{Damping Time Enhancement}

The damping rates, or damping partition numbers, of the collider are constrained according to Robinson's Theorem[5]:

$$
J_{x}+J_{y}+J_{x}=4
$$

where $J_{x}=1-\mathcal{D}, J_{y}=1$, and $J_{s}=2+\mathcal{D}$. The radiation damping times are then given by $\tau_{i}=\tau_{0} / J_{i}$, where $\tau_{0}=$ particle energy/(ave. radiated power). In the above, $\mathcal{D}$ is given by

$$
\mathcal{D}=\frac{\left\langle\frac{D}{\rho^{2}}\left(\frac{1}{\rho}+\frac{2 B^{\prime}}{B}\right)\right\rangle}{\left\langle\frac{1}{\rho^{2}}\right\rangle}
$$

where the averages are taken over the entire ring. For a pure FODO lattice. where $B^{\prime}=0$ when $B$ is not zero, and vice versa. then $J_{x}=J_{y}=1$. and $J_{s}=2$. To take advantage of the synchrotron radiation damping inherent in a $30 \mathrm{TeV}$ proton collider, schemes were investigated to enhance the damping rate. Two lattices consisting of defocusing bending magnets were envisioned. The first lattice used cells containing a single focusing quadrupole and defocusing bending magnets elsewhere. (A design of a combined function lattice for the LHC [6] was reviewed by the working group.) Analytical expressions for this simple system were developed. Though the hardware layout is simple (one quadrupole type, and one bending magnet type), to obtain increased damping one needs a gradient of about $3 \mathrm{~T} / \mathrm{m}$ in a $10 \mathrm{~T}$ magnet. $\left(\mathcal{D} \approx 2\left\langle D B^{\prime} / B\right\rangle=-1\right.$, which for $B=10 \mathrm{~T}$, and $\langle D\rangle=1.5 \mathrm{~m}$ leads to $B^{\prime}=-3 \mathrm{~T} / \mathrm{m}$.) This leads to rather long cells (if we demand $90^{\circ}$ phase advance in both planes) and unacceptable amplitude functions. The second lattice used defocusing bending magnets, but retained both $\mathrm{F}$ and $\mathrm{D}$ quadrupoles in the standard FODO-type structure. In this case, the two quadrupoles are of different strengths (lengths), and so one gives up the simplicity one was after.

Another, simpler scheme involves the misalignment of the arc quadrupoles by roughly $5 \mathrm{~mm}$. If the otherwise standard quadrupole magnets are all moved radially outward by this amount, then the quadrupoles will steer the beam and hence generate radiation. For this case,

$$
\begin{aligned}
\mathcal{D} & =-\left(\frac{8 d}{L \theta_{b}}\right)\left(\frac{L_{b}}{L_{q}}\right) \\
& =-\left(\frac{8 d}{(90 m)(7.5 m r a d)}\right)\left(\frac{80 m}{5 m}\right) \\
& =-1 \\
\rightarrow d & =5.5 \mathrm{~mm},
\end{aligned}
$$

for SSC-type cell parameters. 
The $5.5 \mathrm{~mm}$ shift would thus double the transverse damping rate. On the other hand, it may be more economical or simpler to design a quadrupole magnet with a small central bend field of order $1.2 \mathrm{~T}$ to perform the same task. And, if the quadrupole positions are remotely tunable, as we have previously assumed, then one could contemplate "tuning" the damping rate.

\section{Interaction Regions}

Some comments can be made about general design considerations for Interaction Regions of the $30 \mathrm{TeV}$ accelerator. First of all, much of the work becomes simplified if the two counter-rotating beams have a small separation. If the accelerator used 2-in-1 magnets for instance (as in the LHC) then the problem of bringing the two beams together at the IR, and the associated problem of dispersion suppression, becomes easier. At the workshop, a "simple telescope" design was studied which could generate a $\beta^{*}$ of $20 \mathrm{~cm}$, with a free region on either side of the interaction point of about $20 \mathrm{~m}$. If one could assume a horizontally separated 2-in-1 magnet design, and if the demand for zero slope of the dispersion function at the IP were retracted, then dispersion suppressor modules on either side of the IR could easily be designed to correct the spurious dispersion generated by the crossing and the IR optics becomes rather straightforward. If 2-in-1 magnet systems are used, then the optics of both rings are by default tuned simultaneously. A system of four tuning quadrupoles (three final-focus triplet quads, and a fourth outside the triplet all tunable) can produce the required range of optics for collisions and injection. The $30 \mathrm{TeV}$ ring would require $\approx 400 \mathrm{~T} / \mathrm{m}$ gradient quadrupoles for this design. If 2-in-1 magnets are not adopted, then the IR design may evolve closer to the SSC design, where independent tuning of $\beta^{*}$ can be performed on each ring. [7] If the SSC design were simply scaled to $30 \mathrm{TeV}, \beta^{*}=20 \mathrm{~cm}$ could be produced with similar gradient requirements.

Perhaps the primary problems which will be encountered in the IR design of this accelerator are the same as those which were facing by the SSC - vibration and ground motion, which can cause the beams to miss each other, and energy deposition in the IR magnets. Misalignments of only $0.26 \mu \mathrm{m}$ in a triplet quadrupole can result in a closed orbit motion at the interaction point of one rms beam size. Hence, feedback mechanisms will undoubtedly need to be employed.

The use of non-superconducting magnets in the interaction region were also discussed at the workshop. The use of such devices has the fundamental potential role of protecting the superconducting triplet quads against particle spray from the IP, by acting as collimators. Non-superconducting magnets can also have a much smaller pole tip radius, though a lower pole tip field comes along with them as well. Taking a pole tip radius of $5 \mathrm{~mm}$, for instance, one could acheive gradients of $260 \mathrm{~T} / \mathrm{m}$. A $3 \mathrm{~m}$ long quadrupole of this type, immediately in front of the superconducting triplet can enhance the optical 
performance of the system and simultaneously help to protect the more delicate magnets behind it. While the beam size is easily contained within this aperture, one fear might be that a large betatron oscillation (such as that produced by a kicker misfire in the collider) could cause the beam to strike at this small aperture heading toward, and hence damaging, a detector.

\section{Magnet Aperture Issues}

If full advantage of synchrotron radiation damping enhancements can be realized in this collider - by appropriate choice of lattice, or quadrupole offsets, for example - then the field quality at injection could be relaxed. In this scenario, a smaller magnet bore, or lower injection field might be tolerable. For the Workshop, the assumptions were that the $30 \mathrm{TeV}$ accelerator would use a $1 \mathrm{TeV}$ injector, and would have a fill time of under 15 minutes. For this case it was felt, primarily from SSC experience, that at a minimum $\pm 5 \mathrm{~mm}$ were required for a "good field region," and $\pm 10 \mathrm{~mm}$ of physical aperture was required to perform injection and beam abort procedures. It was felt that the field quality generated by present $50 \mathrm{~mm}$ magnets was more than satisfactory. Because the nonlinear field quality suffers in a minor way by going through the magnet off-axis, one could consider having the $20 \mathrm{~mm}$ beam pipe off-center through the (presumed) $50 \mathrm{~mm}$ magnet bore, if this simplified any engineering efforts of the vacuum/liner designs.

\section{CONCLUDING REMARKS}

The Workshop on Future U.S. Hadron Facilities, held at Indiana University, brought together many interesting ideas of how one may wish to procede with future high energy proton colliders. The goal of $\geq 60 \mathrm{TeV}$ in the center-of-mass, with a luminosity of $1 \times 10^{34}$ is a reasonable goal for such a facility following the LHC. The most interesting and promising factor of this energy regime is the utilization of synchrotron radiation to enhance the accelerator performance, as well as simplifying its construction and thus its cost. A next generation hadron collider of this type certainly deserves attention and further discussion.

Trading off the number of bunches in the accelerator with the number of interactions per bunch crossing has a significant effect on many other accelerator parameters, such as the total stored energy and total synchrotron power absorbed by the superconducting magnets. It is clear that the accelerator and detector designers will need to work closely with each other at arriving at a mutually acceptable set of parameters.

This report did not discuss many details of the technical challenges of such an accelerator, and there will be many. The reader is refered to the Workshop proceedings. [3] Most of the challenges are similar in scope as those 
found in the SSC and LHC designs, such as high field magnets, vacuum and cryogenic cooling in the face of high-flux synchrotron radiation, and so on. But naturally a higher energy device will push the technologies even further. Particle energies in the range of $30 \mathrm{TeV}$ will require bending magnets with fields in the range of $12.5 \mathrm{~T} \pm 3 \mathrm{~T}$. A reinvigorated U.S. superconducting magnet $R \& D$ program will be needed to support these aims.

\section{ACKNOWLEDGEMENTS}

In this presentation, the author is acting the role of rapporteur. The work described and many pieces of text have been performed and/or written by other members of the Workshop. In particular, the author would like to thank Steve Holmes, Mike Harrison, Gerry Dugan, Alex Chao, and Steve Peggs for their contributions, discussions, and leadership during the Workshop.

\section{REFERENCES}

1. "Site-Spec. Concep. Design Rpt. of the SSC," SSCL-SR-1056, 1990.

2. "SSC: A Retrospective Summary - 1989-1993," ed. J. Sanfor : and G. Dugan, SSCL-SR-1235, April 1994.

3. S. Holmes, et al., "Proceedings of the Workshop on Future Hadron Facilites in the U.S.," Fermilab and IUCF Reports, to be published.

4. "Proton - Proton Collider Upgrade Conceptual Design Report," Fermilab, May 1988.

5. K. W. Robinson, "Radiation Effects in Circular Electron Accelerators," Phys. Rev. 111, No. 2 (1958).

6. F. Meot and T. Tortschanoff, "Combined Function Focusing, Combined Function Superconducting Dipole for the LHC," CERN SL/94-20(AP), CERN, 1994.

7. Y. Nosochkov, et al., "Principles of Interaction Region Design in Hadron Colliders and Their Application to the SSC," SSCL-Preprint550, SSCL, 1994. 